\title{
ОЦЕНКА КАЧЕСТВА ЖИЗНИ ПО МЕЖДУНАРОДНОЙ КЛАССИФИКАЦИИ ФУНКЦИОНИРОВАНИЯ ПРИ МНОЖЕСТВЕННЫХ ЦЕРЕБРАЛЬНЫХ АНЕВРИЗМАХ ПОСЛЕ ЭНДОВАСКУЛЯРНОГО ЛЕЧЕНИЯ
}

\author{
А. А. Олейник ${ }^{\square}$, Н. Е. Иванова ${ }^{1}$, Е. А. Олейник ${ }^{1}$, А. Ю. Иванов ${ }^{2,3}$ \\ ${ }^{1}$ Национальный медицинский исследовательский центр имени В. А. Алмазова, Санкт-Петербург, Россия \\ ${ }^{2}$ Санкт-Петербургский государственный педиатрический медицинский университет, Санкт-Петербург, Россия \\ ${ }^{3}$ Северо-Западный государственный медицинский университет имени И. И. Мечникова, Санкт-Петербург, Россия
}

\begin{abstract}
Аневризматическое субарахноидальное кровоизлияние (САК) ассоциировано с высокой летальностью и инвалидизацией больных. Пациенты с множественными церебральными аневризмами (MА) чаще подвержены неоднократным оперативным вмешательствам, у них имеется вероятность образования аневризм de novo и их разрыва. Целью исследования было оценить качество жизни (КЖ) пациентов с помощью Международной классификации функционирования (МКФ) в отдаленном послеоперационном периоде после эндоваскулярного лечения МА для определения пути оптимизации реабилитации. В исследовании приняли участие оперированные эндоваскулярно пациенты с множественными (> 2) церебральными аневризмами (141 человек). Всем пациентам было выполнено 1-6 эндоваскулярных операций, осложнения лечения возникли в 7,1\% (10/141). КЖК определяли в сроки от 6 до 24 месяцев после операции, используя МКФ. Выявлено, что в отдаленном послеоперационном периоде результаты были хуже по доменам, связанным с болью, памятью, координацией движений, силой конечностей. При геморрагическом типе течения результаты по доменам, связанным с передвижением, были хуже по сравнению с другими типами течения ( $p<0,05)$, а при псевдоопухолевом - по доменам, связанным с нарушением функции черепно-мозговых нервов, отвечающих за иннервацию мышц глаза ( $p<0,001)$. По сравнению с показателями дооперационного периода в отдаленном послеоперационном периоде выявлено повышение активности и участия больных с геморрагическим типом течения (p < 0,05). На выраженность нарушений активности влияли осложнения операций, возраст больных ( $p<0,05)$, осложнения острого периода САК ( $p<0,001)$.
\end{abstract}

Ключевые слова: множественные церебральные аневризмы, качество жизни, эндоваскулярное лечение, отдаленные результаты лечения аневризм, оптимизация медицинской реабилитации

Информация о вкладе авторов: А. А. Олейник - сбор, анализ и интерпретация данных, анализ литературы, написание текста статьи; Н. Е. Иванова планирование исследования, редактирование рукописи; Е. А. Олейник — анализ литературы, статистическая обработка; А. Ю. Иванов — редактирование рукописи.

Соблюдение этических стандартов: исследование одобрено этическим комитетом Национального медицинского исследовательского центра имени В. А. Алмазова (протокол № 30 от 13 февраля 2017 г.). Все участники подписали добровольное согласие на участие в исследовании.

$\triangle$ Для корреспонденции: Анна Анатольевна Олейник

ул. Маяковского, д. 12, г. Санкт-Петербург, 191014; doctor.an.an@mail.ru

Статья получена: 19.11.2019 Статья принята к печати: 03.12.2019 Опубликована онлайн: 13.12.2019

DOI: $10.24075 /$ vrgmu.2019.080

\section{QUALITY OF LIFE OF PATIENT WITH MULTIPLE CEREBRAL ANEURYSMS AFTER ENDOVASCULAR TREATMENT: ASSESSMENT BY THE CRITERIA OF INTERNATIONAL CLASSIFICATION OF FUNCTIONING}

Oleynik $A A^{1} \otimes$, Ivanova $N E^{1}$, Oleynik $E A^{1}$, Ivanov $A Y u^{2,3}$

${ }^{1}$ Almazov Medical Research Centre, St. Petersburg, Russia

2 St. Petersburg State Pediatric Medical University, St. Petersburg, Russia

${ }^{3}$ North-Western State Medical University named after I. I. Mechnikov, St. Petersburg, Russia

The rate of mortality and disability associated with aneurysmal subarachnoid hemorrhage (SAH) is high. Patients with multiple cerebral aneurysms (MCA) require repeated surgeries more often and they are likely to develop aneurysms de novo and suffer their rupture. This study aimed to apply the International Classification of Functioning (ICF) to assess the quality of life (QOL) of MCA patients after endovascular treatment, late postoperative period. The study involved patients who underwent endovascular treatment and had multiple (>2) cerebral aneurysms (141 people). All patients underwent 1-6 endovascular surgeries; complications developed in 7.1\% (10/141) of cases. The patients' QOL was assessed against the ICF 6 to 24 months post-surgery. We found that at such time points treatment results deteriorate in a number of domains, namely those associated with pain, memory, motor coordination, limb strength. Patients with ruptured aneurysms showed worse results for locomotion-related domains than patients with unruptured aneurysms $(p<0.05)$, in patients with aneurysms having a pseudotumor type of flow, by domains associated with dysfunction of the cranial nerves responsible for innervation of the eye muscles $(p<0.001)(p<0.001)$. Patients with ruptured MCA were more active in the late post-surgery period, which was revealed by comparing that period's data to the baseline pre-surgery records $(p<0.05)$. The severity of activity disorders depended on surgery complications, patient age $(p<0.05)$, complications that developed during the acute SAH stage $(p<0.001)$.

Keywords: multiple cerebral aneurysms, quality of life, endovascular treatment, late aneurysms treatment results, optimization of medical rehabilitation

Author contribution: Oleynik AA — data collection, analysis and interpretation, literature analysis, article authoring; Ivanova NE — research planning, manuscript editing; Oleynik EA — literature analysis, statistical processing; Ivanov AYu — manuscript editing.

Compliance with ethical standards: the study was approved by the Ethics Committee of the Almazov National Medical Research Centre (minutes \#30 of February 13, 2017). All participants signed a voluntary consent to participate in the study.

$\varangle$ Correspondence should be addressed: Anna A. Oleynik

ul. Mayakovskogo, 12, St. Petersburg, 191014; doctor.an.an@mail.ru

Received: 19.11.2019 Accepted: 03.12.2019 Published online: 13.12.2019

DOI: $10.24075 /$ brsmu.2019.080

Аневризмы сосудов головного мозга - сосудистые аномалии, являющиеся одной из самых частых причин субарахноидального кровоизлияния (САК) [1-2]. За последние
30 лет лечение САК значительно изменилось [3]. Понимание патофизиологических механизмов вазоспазма и ишемии головного мозга после САК привело к улучшению 
интенсивной терапии в остром периоде и уменьшению ишемических осложнений [4-5]. В связи с достижениями хирургии аневризм сосудов головного мозга, в том числе в остром периоде САК, усовершенствованием методов внутрисосудистого выключения аневризм из кровотока уменьшилась доля повторных САК [6]. Развитие и распространение неинвазивной диагностики (магнитнорезонансной ангиографии, спиральной компьютерной ангиографии) привели к обнаружению бессимптомных аневризм сосудов головного мозга [7]. Оценку риска кровоизлияния из аневризмы проводят с учетом морфологических особенностей аневризмы (размера, формы, расположения), а единственным методом лечения остается хирургическое вмешательство [8]. Осложнения оперативных вмешательств на аневризмах варьируют от 3 до 29\% [9-11]. На сегодняшний день, однако, недостаточно исследований качества жизни больных после операции на аневризмах, как бессимптомных, так и проявляющихся кровоизлиянием или псеводоопухолевым течением [12]. Известно, что до 20-30\% больных после перенесенного внутричерепного кровоизлияния остаются инвалидами [13]. Улучшение результатов медицинской реабилитации больных после перенесенного САК, а также ишемических или геморрагических осложнений операций возможно при оптимизации алгоритмов реабилитации с учетом ведущих неврологических нарушений [14]. Оценка качества жизни больных, основанная на Международной классификации функционирования, ограничения жизнедеятельности и здоровья (МКФ), дает целостное представление о различных аспектах здоровья пациента [15]. Цель исследования - оценить качество жизни пациентов с помощью МКФ, в отдаленном послеоперационном периоде после эндоваскулярного лечения множественных аневризм сосудов головного мозга для определения пути оптимизации реабилитационных алгоритмов.

\section{ПАЦИЕНТЫ И МЕТОДЫ}

В исследование были включены пациенты с множественными аневризмами сосудов головного мозга (141 человек), получившие эндоваскулярное лечение в РНХИ имени
А. Л. Поленова в 2010-2018 гг. Среди них лиц мужского пола было 17\% (24/141), лиц женского пола — 83\% (117/141); средний возраст пациентов составил 54,16 \pm 11,24 года. Критерии включения пациентов в исследование: наличие у пациента множественных (> 2) церебральных аневризм, оперированных эндоваскулярно; возможность проследить у больного отдаленные результаты. Критерии исключения: возраст меньше 18 лет; наличие у больного аневризм в сочетании с артериовенозными мальформациями; наличие в анамнезе микрохирургического лечения аневризмы. В каждом наблюдении было диагностировано от 2 до 6 аневризм: 2 аневризмы были выявлены в 62,4\% случаев (88/141), 3 аневризмы - в 26,2\% (36/141), 4 аневризмы - в 7,8\% (11/141), 5 аневризм - в 2,8\% (4/141), 6 аневризм — в 0,7\% (1/141). В 141 наблюдении 349 аневризм. Размеры аневризм: миллиарные (до 3 мм) 22,9\% (80/349), обычного размера (4-15 мм) - 67,9\% (237/349), большие (16-25 мм) - 5,2\% (18/349), гигантские (> 25 мм) - 4,0\% (14/349). В дооперационном периоде перенесли одно или несколько субарахноидальных кровоизлияний - 45,4\% пациентов (64 наблюдения), имели псевдоопухолевый (проявляющийся объемным воздействием на окружающие структуры) тип течения аневризмы - 7,1\% (10 наблюдений), бессимптомный тип течения (случайно выявленные аневризмы) - 47,5\% (67 наблюдений). Всем пациентам выполнено от 1 до 6 эндоваскулярных операций (всего 271 операция): эндоваскулярная окклюзия аневризм отделяемыми спиралями - 42,4\% (115/271), эндоваскулярная окклюзия аневризм отделяемыми спиралями с использованием ассистирующих методик (стент- и баллон-ассистенция) 32,8\% (89/271), установка поток-отклоняющего стента 24,7\% (67/271). Осложнения эндоваскулярного лечения (вазоспазм, ишемические осложнения, геморрагические осложнения) возникали в 7,1\% случаев (10/141), из них стойкий неврологический дефицит развился в 4,3\% (6/141). Качество жизни определяли в отдаленном послеоперационном периоде в сроки от 6 до 24 месяцев. Для этих целей использовали МКФ [15]. Оценивали домены нарушения функции организма, домены активности и участия (табл. 1).

Таблица 1. Домены нарушения функции, активности и участия и методы их оценки

\begin{tabular}{|l|l|}
\hline \multicolumn{1}{|c|}{ Домены нарушения функции организма } & \multicolumn{1}{|c|}{ Метод оценки } \\
\hline b144 Функции памяти & МMSE [16] \\
\hline b2152 Функции мышц глаза & $\begin{array}{l}\text { Изолированная оценка функции мышц глаза по шкале повреждения } \\
\text { функциональных систем по J. F. Кurtzke* }\end{array}$ \\
\hline b2702 Тактильная чувствительность & Шкальная оценка* \\
\hline b167 Умственные функции речи & Шкала нарушения речи Л. И. Вассермана [17] \\
\hline b28010 Боль в голове и шее & Визуальная аналоговая шкала оценки боли [18] \\
\hline b320 Функции артикуляции & Оценка по степени тяжести \\
\hline b730 Функции мышечной силы & Шкала оценки мышечной силы \\
\hline b7602 Координация произвольных движений & GET UP АND GO ТEST [19] \\
\hline d450 Ходьба & Тест шестиминутной ходьбы [20] \\
\hline d640 Выполнение работы по дому & Шкала Рэнкина [21], расширенная шкала исходов Глазго [22] \\
\hline d510 Мытье & Индекс повседневной активности Бартел [23], шкала Рэнкина \\
\hline d540 Одевание & Индекс повседневной активности Бартел \\
\hline d550 Прием пищи & Индекс повседневной активности Бартел \\
\hline
\end{tabular}

Примечание: * - 0 - нет изменений; 1 - симптомы без нарушения функций (легкие нарушения, выявляемые при неврологическом осмотре, у пациента жалоб нет); 2 - легкие нарушения (легкое нарушение движений глаз, пациент жалуется на двоение, имеется парез какой-либо одной наружной мышцы глаза); 3 - умеренные нарушения (умеренные нарушения движений глаз, у пациента есть жалобы на двоение); 4 - выраженные нарушения (паралич одной или нескольких наружных мышц глаза); ${ }^{\star}$ — 0 - нет нарушений; 1 - легкие нарушения; 2 - умеренные нарушения; 3 - тяжелые нарушения; 4 абсолютные нарушения. 
Bce данные заносили в электронную таблицу Microsoft Excel 7.0. Полученные в исследовании клинические данные обрабатывали в STATISTICA for Windows 10.0 (StatSoft, Tulsa; USA). Сравнение количественных параметров (оценку по доменам МКФ, шкале Рэнкина, расширенной шкале исходов Глазго, индекса повседневной активности Бартел в зависимости от возрастных групп, наличия осложнений САК, осложнений оперативных вмешательств, групп дооперационного клинического течения) проводили с помощью критериев Манна-Уитни, медианного $\chi^{2}$ и модуля ANOVA. Динамику показателей по доменам активности и участия до и после оперативного вмешательства оценивали с помощью критерия Уилкоксона. Статистически достоверными считали выводы при величине $p<0,05$.

\section{РЕЗУЛЬТАТЫ ИССЛЕДОВАНИЯ}

Оценка неврологической симптоматики и жалоб пациента в отдаленном послеоперационном периоде представлена на рис. 1. Оценка доменов активности и участия в определителе «капаситет» и «реализация» представлена на рис. 2.

В связи с тем, что МКФ позволяет системно оценить состояние нарушений функций организма, используя единую оценку степени выраженности нарушений, было выявлено, что чаще показатели были хуже по доменам, связанным с болью (b28010), функциям памяти (b144), несколько реже - с координацией движений (b7602), силой мышц (b7302). По разделу активности и участия чаще были выявлены нарушения, связанные с ходьбой (d450), выполнением работы по дому (d640).

При оценке выявленных нарушений в отдаленном послеоперационном периоде в зависимости от типа предоперационного течения аневризм обнаружено, что при геморрагическом типе течения результаты по доменам, связанным с передвижением (b 7302, d4602, d640), были достоверно хуже по сравнению с другими типами течения множественных аневризм $(p<0,05)$. Нарушения функций черепно-мозговых нервов, отвечающих за иннервацию мышц глаза (III, IV, VI), достоверно чаще были в группе с псевдоопухолевым типом течения аневризм, даже в отдаленном послеоперационном периоде $(p<0,001)$. Достоверной разницы между группами в других доменах выявлено не было ( $p>0,05)$. Оценка цефалгического синдрома после операции показала, что нет статистически значимой разницы у пациентов с разорвавшимися и неразорвавшимися аневризмами. Данный факт указывает на значимость детального изучения структуры цефалгического синдрома и его причины.

При сравнении дооперационных данных и полученных в отдаленном послеоперационном периоде выявлено улучшение качества жизни больных с геморрагическим типом течения по доменам d4602 (передвижение вне своего дома и вне других зданий; рис. 3) ( $(p=0,004)$, d640 (выполнение работы по дому) ( $p=0,03)$.

На степень выраженности нарушений активности (оценка с помощью индекса повседневной активности Бартел, шкалы Рэнкина, расширенной шкалы исходов Глазго) оказывали вляние осложнения после оперативных вмешательств $(p<0,05)$, возраст больных от 51 до 60 лет ( $p<0,05)$, осложнения острого периода САК ( $p<0,001)$.

В отдаленном послеоперационном периоде были выявлены следующие осложнения: разрыв аневризмы - 1,4\% (2/141, с формированием очаговой неврологической симптоматики), тромбоз сосуда 2,1\% (3/141, в одном наблюдении с формированием очаговой неврологической симптоматики). В связи с наличием неоперированной аневризмы, проявившей

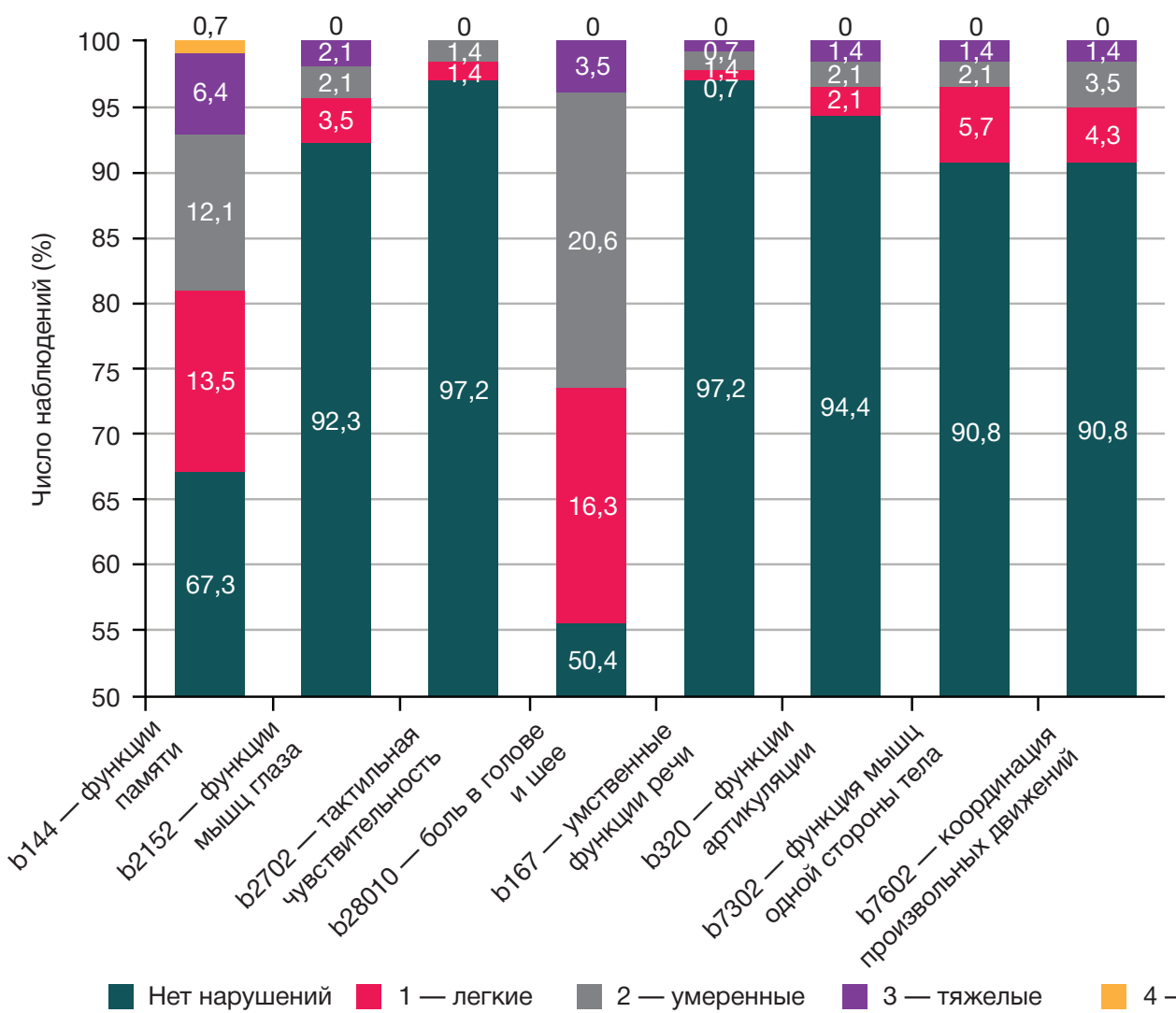

Рис. 1. Оценка по доменам нарушений функций организма у больных с множественными церебральными аневризмами в отдаленном послеоперационном Рис. 1. Оц
периодах 
себя внутричерепным кровоизлиянием, больные были направлены на хирургическое лечение с последующими реабилитационными мероприятиями.

Таким образом, оценка степени нарушений по МКФ позволяет определять пути реабилитации таких больных.

\section{ОБСУЖДЕНИЕ РЕЗУЛЬТАТОВ}

По результатам нашего исследования, у пациентов как с одиночными, так и с множественными аневризмами, наличие в анамнезе субарахноидального кровоизлияния снижает функциональные результаты, что подтверждают и литературные данные [24-26]. Как и в нашем исследовании, в ряде других работ были выявлены следующие факторы, влияющие на функциональный исход: возраст больных на момент кровоизлияния [24], паренхиматозное кровоизлияние [24-25], большой и гигантский размер аневризмы [25]. Полученные нами данные расходятся с результатами других исследований, выделяющих из факторов также локализацию аневризмы в области средней мозговой артерии [24, 30], внутрижелудочковое кровоизлияние [27].

В последние годы принято использовать МКФ для оценки результатов лечения и/или реабилитации при заболеваниях и повреждениях нервной системы [28] и других систем организма [29]. Однако работ, посвященных оценке результатов эндоваскулярного лечения больных с множественными аневризмами с помощью МКФ, недостаточно. Так, с использованием МКФ были классифицированы детерминанты при обобщении

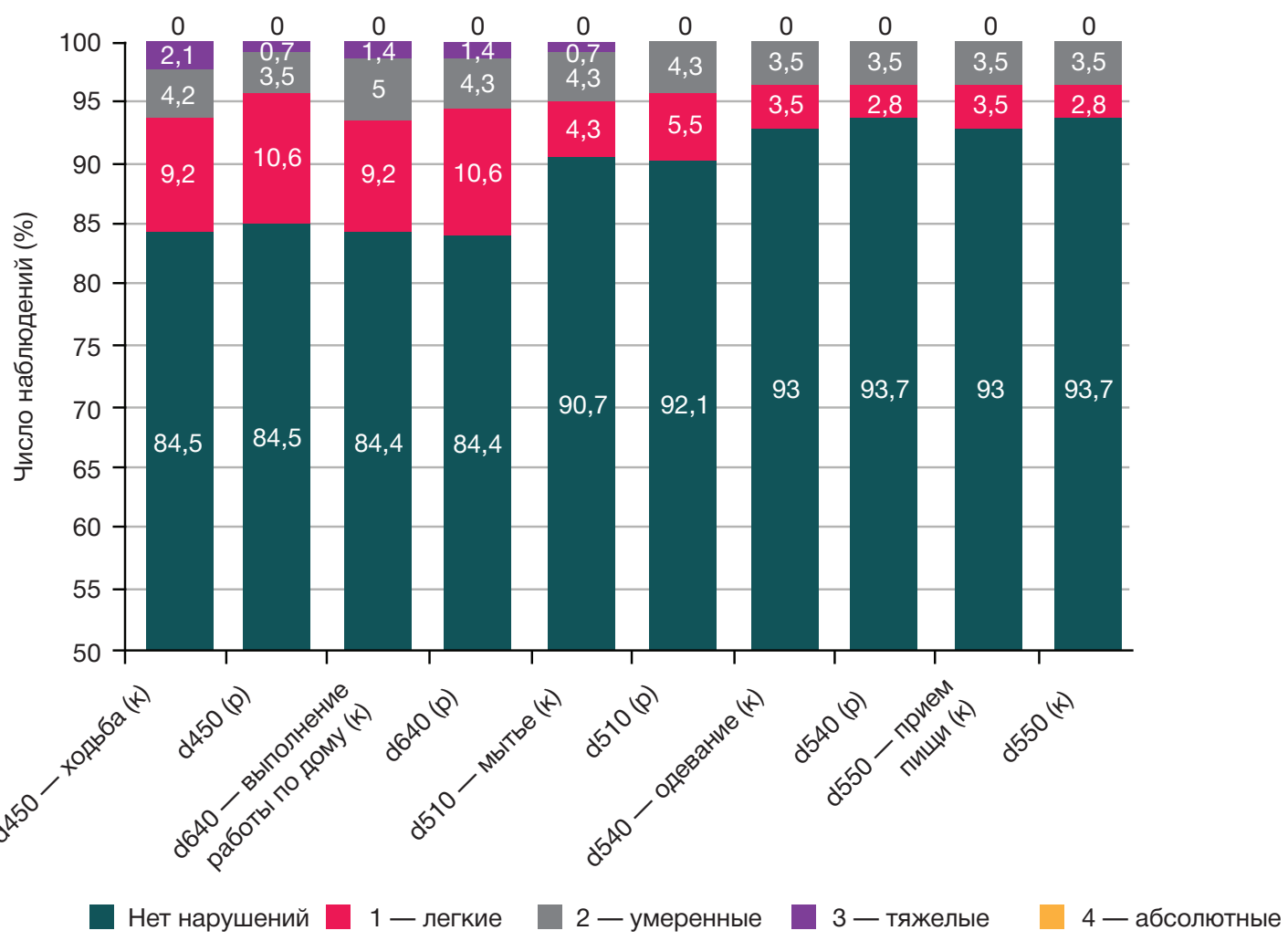

Рис. 2. Оценка по доменам активности и участия в определителе «капаситет» (к) и «реализация» (р) у больных с множественными церебральными аневризмами в отдаленном послеоперационном периоде

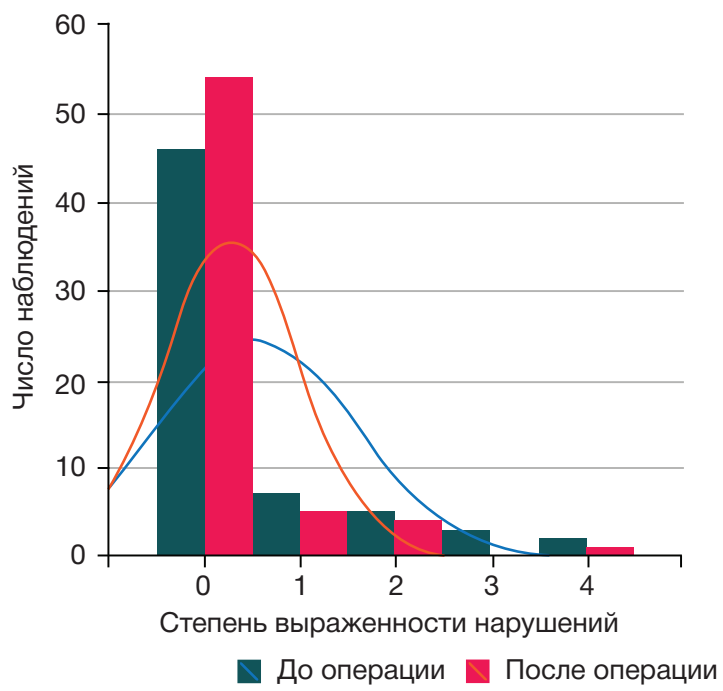

Рис. 3. Оценка по домену d4602 (передвижение вне своего дома и вне других зданий) у больных с геморрагическим типом течения дооперационно и в отдаленном послеоперационном периоде 
данных разных исследований по детерминантам снижения качества жизни у больных после аневризматического субарахноидального кровоизлияния [30]. Было выявлено, что детерминанты, связанные со структурой и функциями организма (клиническое состояние при поступлении, снижение настроения), ограничения активности (физическая инвалидность и когнитивные жалобы) и личностные факторы (женский пол, более старший возраст) связаны с ухудшением качества жизни после аневризматического САК. В нашем исследовании тоже показано снижение качества жизни у больных с нарушениями функций организма (b7302, b2152).

\section{ВЫВОДЫ}

В отдаленном послеоперационном периоде после эндоваскулярного лечения множественных церебральных аневризм качество жизни снижено у больных, перенесших CAK, а также у имеющих крупные аневризмы с псеводоопухолевым типом течения. В связи с вероятностью образования аневризм de novo, а также реканализации аневризм, имеется риск повторного САК. Таким образом, дальнейшие реабилитационные мероприятия необходимо проводить с учетом нейровизуализационных данных в отдаленном послеоперационном периоде. Оценка состояния пациента по МКФ позволяет описать нарушения функций организма, его активности и участия, формулировать цели реабилитации, оценивать результаты эндоваскулярного лечения и дальнейших реабилитационных мероприятий. Использование отдельных шкал препятствует проведению системного анализа состояния пациента. Единое описание степени выраженности нарушений не только удобно для оценки результатов, но и позволяет проводить научные исследования, выполнять их сравнение друг с другом.

\section{Литература}

1. Крылов В. В., редактор. Хирургия аневризм головного мозга. В трех томах. Том І. М.: Изд-во ИП «Т. А. Алексеева», 2011; $432 \mathrm{c}$.

2. Азаров М. В., Купатадзе Д. Д., Набоков В. В. Синдром Клиппеля-Треноне, этиология, патогенез, диагностика и лечение. Педиатрия. 2018; 9 (2): 78-86.

3. La Pira B, Singh TD, Rabinstein AA, Lanzino G. Time Trends in Outcomes After Aneurysmal Subarachnoid Hemorrhage Over the Past 30 Years. Mayo Clin Proc. 2018 Dec; 93 (12): 1786-93.

4. Diringer MN, Zazulia AR. Aneurysmal Subarachnoid Hemorrhage: Strategies for Preventing Vasospasm in the Intensive Care Unit. Semin Respir Crit Care Med. 2017 Dec; 38 (6): 760-7.

5. Горощенко С. А., Иванова Н. Е., Рожченко Л. В., Забродская Ю. М., Размологова А. Ю., Кондратьев А. Н. и др. Экстрапонтинный миелинолиз, развившийся после аневризматического субарахноидального кровоизлияния (случай из практики и обзор литературы). Вопросы нейрохирургии. 2016; (6): 82-7.

6. Anxionnat R, Tonnelet R, Derelle AL, Liao L, Barbier C, Bracard S. Endovascular treatment of ruptured intracranial aneurysms: Indications, techniques and results. Diagn Interv Imaging. 2015 Jul-Aug; 96 (7-8): 667-75.

7. Крылов В. В., Элиава Ш. Ш., Яковлев С. Б., Хейреддин А. С., Белоусова О. Б., Полунина Н. А. Клинические рекомендации по лечению неразорвавшихся бессимптомных аневризм головного мозга. Вопросы нейрохирургии. 2016; 80 (5): $124-35$.

8. Wiebers DO, Whisnant JP, Huston J 3rd, Meissner I, Brown RD Jr, Piepgras DG, et al. International Study of Unruptured Intracranial Aneurysms Investigators. Unruptured intracranial aneurysms: natural history, clinical outcome, and risks of surgical and endovascular treatment. Lancet. 2003 Jul 12; 362 (9378): 103-10.

9. Ihn YK, Shin SH, Baik SK, Choi IS. Complications of endovascular treatment for intracranial aneurysms: Management and prevention. Interv Neuroradiol. 2018 Jun; 24 (3): 237-45.

10. Oishi H, Yamamoto M, Shimizu T, Yoshida K, Arai H. Endovascular therapy of 500 small asymptomatic unruptured intracranial aneurysms. AJNR Am J Neuroradiol. 2012 May; 33 (5): 958-64.

11. Kim DY, Park JC, Kim JK, Sung YS, Park ES, Kwak JH, Choi CG, Lee DH. Microembolism after Endovascular Treatment of Unruptured Cerebral Aneurysms: Reduction of its Incidence by Microcatheter Lumen Aspiration. Neurointervention. 2015 Sep; 10 (2): 67-73

12. Dammann $P$, Wittek $P$, Darkwah Oppong $M$, Hütter $B O$, Jabbarli $R$, et al. Relative health-related quality of life after treatment of unruptured intracranial aneurysms: long-term outcomes and influencing factors. Ther adv neurol disord. 2019; (12): 1-12.
13. le Roux AA, Wallace MC. Outcome and cost of aneurysmal subarachnoid hemorrhage. Neurosurg Clin N Am. 2010 Apr; 21 (2): 235-46.

14. Geyh S, Cieza A, Schouten J, Dickson H, Frommelt P, Omar Z, et al. ICF Core Sets for stroke. J Rehabil Med. 2004 Jul; (44 Suppl): 135-41.

15. World Health Organization. International Classification of Functioning, Disability and Health: ICF. Geneva: WHO; 2001.

16. Folstein MF, Folstein SE, McHugh PR. «Mini-mental state» a practical method for grading the cognitive state of patients for the clinician. Journal of Psychiatric Research. 1975; 12 (3): 189-98.

17. Вассерман Л. И., Дорофеева С. А., Меерсон Я. А. Методы нейропсихологической диагностики. Практическое руководство СПб.: Стройлеспечать, 1997.

18. Scott J, Huskisson EC. Graphic representation of pain. Pain 1976; 2 (2): 175-84.

19. Mathias S, Nayak US, Isaacs B. Balance in elderly patients: the "get-up and go" test. Arch Phys Med Rehabil. 1986 Jun; 67 (6): 387-9.

20. American Thoracic Society statement: guidelines for the sixminute walk test. Am J Respir Crit Care Med. 2002; 166 (1): 111-7.

21. Bonita R, Beaglehole R. Modification of Rankin Scale: Recovery of motor function after stroke. Stroke. 1988 Dec; 19 (12): 1497-500.

22. Wilson JT, Pettigrew LE, Teasdale GM. Structured interviews for the Glasgow Outcome Scale and the extended Glasgow Outcome Scale: guidelines for their use. J Neurotrauma. 1998; (15): 573-85.

23. Mahoney F. Barthel D. Functional evaluation: the Barthel Index. Md Med J. 1965; (14): 61-5.

24. Preiss M, Netuka D, Koblihova J, Bernardova L, Charvat F, Kratochvilova D, et al. Quality of life 1 year after aneurysmal subarachnoid hemorrhage in good outcome patients treated by clipping or coiling. J Neurol Surg A Cent Eur Neurosurg. 2012 Aug: 73 (4): 217-23.

25. Andersen CR, Fitzgerald E, Delaney A, Finfer S. Systematic Review of Outcome Measures Employed in Aneurysmal Subarachnoid Hemorrhage (aSAH). Clin res neurocritical care. 2019; 30 (3): 534-41.

26. AlMatter M, Aguilar Péreza M, Bhogal P, Hellstern V, Ganslandt $O$, Henkes H. Results of interdisciplinary management of 693 patients with aneurysmal subarachnoid hemorrhage: Clinical outcome and relevant prognostic factors. Clin Neurol Neurosurg. 2018 Apr; (167): 106-11.

27. Visser-Meily JMA, Rhebergen ML, Rinkel GJE, van Zandvoort MJ, Post MWM. Long-term health related quality of life after aneurysmal subarachnoid hemorrhage; relationship with psychological symptoms and personality characteristics. Stroke. 2009; (40): 1526-9. 
28. Бодрова Р. А., Аухадеев Э. И., Тихонов И. В. Опыт применения международной классификации функционирования в оценке эффективности реабилитации пациентов с последствиями поражения ЦНС. Практическая медицина. 2013; 1 (66): 98-100.

29. Пономаренко Г. Н., Шошмин А. В., Бесстрашнова Я. К., Черкашина И. В. Планирование и оценка эффективности реабилитации больных остеоартрозом: использование базового

\section{References}

1. Krylov W, redaktor. Hirurgija anevrizm golovnogo mozga. V treh tomah. Tom I. M.: Izd-vo IP «T. A. Alekseeva», 2011; 432 s. Russian.

2. Azarov MV, Kupatadze DD, Nabokov VV. Sindrom KlippeljaTrenone, jetiologija, patogenez, diagnostika i lechenie. Pediatrija. 2018; 9 (2): 78-86. Russian.

3. La Pira B, Singh TD, Rabinstein AA, Lanzino G. Time Trends in Outcomes After Aneurysmal Subarachnoid Hemorrhage Over the Past 30 Years. Mayo Clin Proc. 2018 Dec; 93 (12): 1786-93.

4. Diringer MN, Zazulia AR. Aneurysmal Subarachnoid Hemorrhage: Strategies for Preventing Vasospasm in the Intensive Care Unit. Semin Respir Crit Care Med. 2017 Dec; 38 (6): 760-7.

5. Goroshhenko SA, Ivanova NE, Rozhchenko LV, Zabrodskaja YuM, Razmologova AYu, Kondratev AN, i dr. Jekstrapontinnyj mielinoliz, razvivshijsja posle anevrizmaticheskogo subarahnoidal'nogo krovoizlijanija (sluchaj iz praktiki i obzor literatury). Voprosy nejrohirurgii. 2016; (6): 82-7. Russian.

6. Anxionnat R, Tonnelet R, Derelle AL, Liao L, Barbier C, Bracard S. Endovascular treatment of ruptured intracranial aneurysms: Indications, techniques and results. Diagn Interv Imaging. 2015 Jul-Aug; 96 (7-8): 667-75.

7. Krylov W, Eliava ShSh, Yakovlev SB, Kheyreddin AS, Belousova OB, Polunina NA. Klinicheskie rekomendacii po lecheniju nerazorvavshihsja bessimptomnyh anevrizm golovnogo mozga. Voprosy nejrohirurgii. 2016; 80 (5): 124-35. Russian.

8. Wiebers DO, Whisnant JP, Huston J 3rd, Meissner I, Brown RD Jr, Piepgras DG, et al. International Study of Unruptured Intracrania Aneurysms Investigators. Unruptured intracranial aneurysms: natural history, clinical outcome, and risks of surgical and endovascular treatment. Lancet. 2003 Jul 12; 362 (9378): 103-10.

9. Ihn YK, Shin SH, Baik SK, Choi IS. Complications of endovascular treatment for intracranial aneurysms: Management and prevention. Interv Neuroradiol. 2018 Jun; 24 (3): 237-45.

10. Oishi H, Yamamoto M, Shimizu T, Yoshida K, Arai H. Endovascular therapy of 500 small asymptomatic unruptured intracranial aneurysms. AJNR Am J Neuroradiol. 2012 May; 33 (5): 958-64.

11. Kim DY, Park JC, Kim JK, Sung YS, Park ES, Kwak JH, Choi CG, Lee DH. Microembolism after Endovascular Treatment of Unruptured Cerebral Aneurysms: Reduction of its Incidence by Microcatheter Lumen Aspiration. Neurointervention. 2015 Sep; $10(2): 67-73$

12. Dammann $P$, Wittek $P$, Darkwah Oppong $M$, Hütter $B O$, Jabbarli $R$, et al. Relative health-related quality of life after treatment of unruptured intracranial aneurysms: long-term outcomes and influencing factors. Ther adv neurol disord. 2019; (12): 1-12.

13. le Roux AA, Wallace MC. Outcome and cost of aneurysmal subarachnoid hemorrhage. Neurosurg Clin N Am. 2010 Apr; 21 (2): 235-46.

14. Geyh S, Cieza A, Schouten J, Dickson H, Frommelt P, Omar Z, et al. ICF Core Sets for stroke. J Rehabil Med. 2004 Jul; (44 Suppl): 135-41.

15. World Health Organization. International Classification of Functioning, Disability and Health: ICF. Geneva: WHO; 2001.

16. Folstein MF, Folstein SE, McHugh PR. «Mini-mental state» a набора Международной классификации функционирования, ограничений жизнедеятельности и здоровья. Вопросы курортологии, физиотерапии и лечебной физической культуры. 2017; (1): 4-8.

30. Passier PE, Visser-Meily JM, Rinkel GJ, Lindeman E, Post MW. Determinants of health-related quality of life after aneurysmal subarachnoid hemorrhage: a systematic review. Qual Life Res. 2013 Jun; 22 (5): 1027-43.

practical method for grading the cognitive state of patients for the clinician. Journal of Psychiatric Research. 1975; 12 (3): 189-98.

17. Vasserman LI, Dorofeeva SA, Meerson YaA. Metody nejropsihologicheskoj diagnostiki. Prakticheskoe rukovodstvo. SPb.: Strojlespechat', 1997. Russian.

18. Scott J, Huskisson EC. Graphic representation of pain. Pain. 1976; 2 (2): 175-84.

19. Mathias S, Nayak US, Isaacs B. Balance in elderly patients: the "get-up and go" test. Arch Phys Med Rehabil. 1986 Jun; 67 (6): 387-9.

20. American Thoracic Society statement: guidelines for the sixminute walk test. Am J Respir Crit Care Med. 2002; 166 (1): 111-7.

21. Bonita R, Beaglehole R. Modification of Rankin Scale: Recovery of motor function after stroke. Stroke. 1988 Dec; 19 (12): 1497-500.

22. Wilson JT, Pettigrew LE, Teasdale GM. Structured interviews for the Glasgow Outcome Scale and the extended Glasgow Outcome Scale: guidelines for their use. J Neurotrauma. 1998; (15): 573-85.

23. Mahoney F, Barthel D. Functional evaluation: the Barthel Index. Md Med J. 1965; (14): 61-5.

24. Preiss M, Netuka D, Koblihova J, Bernardova L, Charvat F, Kratochvilova D, et al. Quality of life 1 year after aneurysmal subarachnoid hemorrhage in good outcome patients treated by clipping or coiling. J Neurol Surg A Cent Eur Neurosurg. 2012 Aug; 73 (4): 217-23.

25. Andersen CR, Fitzgerald E, Delaney A, Finfer S. Systematic Review of Outcome Measures Employed in Aneurysmal Subarachnoid Hemorrhage (aSAH). Clin res neurocritical care. 2019; 30 (3): 534-41.

26. AlMatter M, Aguilar Péreza M, Bhogal P, Hellstern V, Ganslandt O, Henkes H. Results of interdisciplinary management of 693 patients with aneurysmal subarachnoid hemorrhage: Clinical outcome and relevant prognostic factors. Clin Neurol Neurosurg. 2018 Apr; (167): 106-11.

27. Visser-Meily JMA, Rhebergen ML, Rinkel GJE, van Zandvoort MJ, Post MWM. Long-term health related quality of life after aneurysmal subarachnoid hemorrhage; relationship with psychological symptoms and personality characteristics. Stroke. 2009; (40): 1526-9.

28. Bodrova RA, Auhadeev Yel, Tihonov IV. Opyt primenenija mezhdunarodnoj klassifikacii funkcionirovanija $v$ ocenke jeffektivnosti reabilitacii pacientov $s$ posledstvijami porazhenija CNS. Prakticheskaja medicina. 2013; 1 (66): 98-100. Russian.

29. Ponomarenko GN, Shoshmin AV, Besstrashnova YaK, Cherkashina IV. Planirovanie i ocenka jeffektivnosti reabilitacii bol'nyh osteoartrozom: ispol'zovanie bazovogo nabora Mezhdunarodnoj klassifikacii funkcionirovanija, ogranichenij zhiznedejatel'nosti i zdorov'ja. Voprosy kurortologii, fizioterapii i lechebnoj fizicheskoj kul'tury. 2017; (1): 4-8. Russian.

30. Passier PE, Visser-Meily JM, Rinkel GJ, Lindeman E, Post MW Determinants of health-related quality of life after aneurysmal subarachnoid hemorrhage: a systematic review. Qual Life Res. 2013 Jun; 22 (5): 1027-43. 